\title{
Trajectory Tracking Control of Underactuated USV with Model Perturbation and External Interference
}

\author{
Jinbiao Liu , Jun Luo ${ }^{1,2}$, Jianxiang Cui, Yan Peng ${ }^{3}$ \\ Department of Mechatronic Engineering Shanghai University, Shanghai, China
}

\begin{abstract}
This paper presents an improved trajectory tracking controller based backstepping control algorithm to address the trajectory tracking problem of an underactuated Unmanned Surface Vessel (USV). A Three Degree of Freedom (DOF) underactuated maneuvering motion model for water-jet-propelled USV is established, and controller can track both straight line trajectory and curve trajectory with relatively high accuracy. The stability of USV system is proved by BIBO (bounded-input-bounded-output stable ) characteristics, then simulation experiments are carried out to demonstrate the robustness and precise control performance of the controller.
\end{abstract}

\section{Introduction}

These Unmanned Surface Vessel (USV), also known as the water robot, which is an unmanned surface vehicle with autonomous planning and navigation capability. Generally, the main difficulties in the tracking control of USV are: system uncertainty, respectively, uncertainty within the system and external uncertainty of the system; underactuated characteristic of the system, the mainstream USV only fits with a water jet propeller, there is no horizontal drive, belong to a typical under-actuated system. how to make the system of USV in high-speed movement to maintain the desired tracking accuracy while maintaining maneuverability has become a hot research topic.

In recent years, the track control of USV has attracted wide attention in the academic field. At home and abroad, there is a deep research on the track control of the USV, and there are many kinds of research methods, such as PID and improved PID, Lyapunov direct method, Backstepping design method,etc. Godhavn et al. [1] designed a global exponential stabilization controller which can achieve good tracking performance in the external environment by using the Backstepping design method and feedback linearization, but the heading angle under the controller is not controlled. Pettersen et al. [2] designed a trajectory tracker which can make underactuated ship's heading tracking error and position tracking error achieve exponential stability on the assumption that the expected heading angle acceleration is greater than zero conditions. K. Do et al. [3] designed a

trajectory tracking controller for under-actuated ship base on the Lyapunov direct method, the backstepping method and the cascade system theory, the controller can finish straight and curved global asymptotic tracking control.

In view of the USV have problems that model parameters uncertainty effect the USV path tracking, A.P. Aguiar et al. [4] combined the logic switch control with Backstepping technology, and designed the adaptive supervisory controller, this method made the path tracking error converges to any small neighborhood of the origin, the simulation results proved the effectiveness of the controller. Peng et al. [5] used the Calman filter to estimate uncertain parameters and designed the path tracking controller. In order to solve the adverse effects of ship model uncertainty and external disturbance ,Do et al. [6] proposed a robust adaptive controller base on Lyapunov direct method and Backstepping after carrying out non-linear coordinate transformation, and estimated the uncertainty of model parameters by using the Lipschitz parameter projection technique.

Though researchers have made lots of contributions and proposed many pioneering methods for trajectory tracking control of USV in the literature mentioned above, in the face of complex sea conditions, the tracking control accuracy is still unable to reach the ideal level. This paper aims to provide and prove a nonlinear backstepping trajectory tracking method which can track an arbitrary reference trajectory for underactuated USV.

\section{Problem formulation}

\footnotetext{
${ }^{1}$ Fund program: Science and Technology Commission of Shanghai Municipality, China(Grant No. 14DZ1110900)

${ }^{2}$ Fund program: Project of Shanghai outstanding academic leader,China(Grant No. 15XD1501800)

${ }^{3}$ Fund program: Supported by Natural Science Foundation of Shanghai Municipality, China(Grant No.

13ZR1454300)
} 


\subsection{USV modeling}

Ships are complex motion with 6-DOF in the sea, including model parameters perturbation for the hull and the complexity of external environment interference, it's difficult to establish accurate model of 6-DOF for USV. Therefore, the research is mainly considering the movement of the surge, sway and yaw directions on the horizontal plane, ignoring the effects of heave, pitch and roll motion, only about 3-DOF motion model. Inertial reference coordinate system $O_{E}-X_{E} Y_{E} Z_{E} \quad$ with origin defined on Earth and body-fixed reference $O_{\mathrm{b}}-X_{\mathrm{b}} Y_{\mathrm{b}} Z_{\mathrm{b}}$ are established as show in Fig. 1. the mathematical model of an underactuated USV moving in the horizontal plane can be described as follows[7]:

$$
\left\{\begin{array}{l}
\dot{\eta}=J(\eta) v \\
M \dot{v}=-C(v)-D(v) v+\tau
\end{array}\right.
$$

with

$$
\begin{gathered}
\eta=[x, y, \psi]^{T}, v=[u, v, r]^{T}, \tau=\left[\tau_{1}, \tau_{2}, \tau_{3}\right]^{T}, \\
M=\operatorname{diag}\left\{m_{11} m_{22} m_{33}\right\}, D(v)=\operatorname{diag}\left\{d_{11} d_{22} d_{33}\right\} \\
J(\psi)=\left(\begin{array}{ccc}
\cos (\psi) & -\sin (\psi) & 0 \\
\sin (\psi) & \cos (\psi) & 0 \\
0 & 0 & 1
\end{array}\right) \\
C(v)=\left(\begin{array}{ccc}
0 & 0 & -m_{22} v \\
0 & 0 & -m_{11} \mu \\
m_{22} v & m_{11} \mu & 0
\end{array}\right)
\end{gathered}
$$

where $(x, y)$ are the Cartesian coordinated of USV's center of mass in $O_{E}-X_{E} Y_{E} Z_{E}, \psi$ denotes yaw angle in $O_{\mathrm{b}}-X_{\mathrm{b}} Y_{\mathrm{b}} Z_{\mathrm{b}} \cdot \mu, v, r$ denote surge, sway and yaw velocities expressed in $O_{\mathrm{b}}-X_{\mathrm{b}} Y_{\mathrm{b}} Z_{\mathrm{b}}$, $\left[\tau_{1}, \tau_{2}, \tau_{3}\right]^{T}$ are control inputs of USV, $\tau_{2}=0$, $m_{11}, m_{22}, m_{33}$ are USV's inertia coefficients including added mass effects, $d_{11}, d_{22}, d_{33}$ are hydrodynamic damping coefficients.

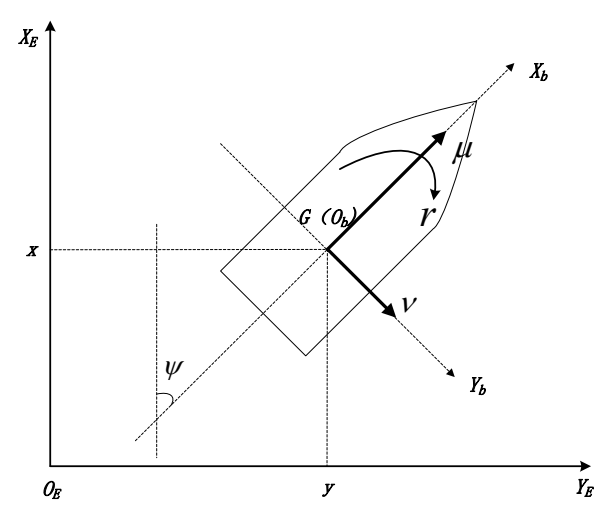

Figure 1. Inertial reference frame and body-fixed reference.
By the above formula can get the kinematics equation of the USV:

$$
\left\{\begin{array}{l}
\dot{x}=u \cos (\psi)-v \sin (\psi) \\
\dot{y}=u \sin (\psi)-v \sin (\psi) \\
\dot{\psi}=r
\end{array}\right.
$$

Without considering the environmental disturbance, the dynamic equation of the USV can be expressed as:

$$
\left\{\begin{array}{l}
m_{11} \dot{u}-m_{22} u r+d_{11} u=\tau_{1} \\
m_{22} \dot{v}+m_{11} u r+d_{22} v=0 \\
m_{33} \dot{r}+\left(m_{22}-m_{11}\right) u v+d_{33} r=\tau_{3}
\end{array}\right.
$$

where $\tau_{1}$ is longitudinal driving force for the control input, $\tau_{3}$ is yaw moment generated by the combined action of propeller and rudder.

\subsection{Problem statement}

In this paper, the control problem of trajectory tracking for USV can be formulated as follows: Considering the situation that model parameter perturbation and external disturbance factors which exist in underactuated Unmanned Surface Vessel (USV), robust control is the key problem of the USV, and the control objective for trajectory tracking of USV is to ensure the tracking errors converge to an arbitrarily small neighborhood of zero as $t$ $\rightarrow \infty$.

\section{Controller design}

\subsection{Sliding mode control law design}

Suppose an expected track of USV:

$$
\left\{\begin{array}{l}
\dot{x}_{d}=u_{d} \cos \left(\psi_{d}-v_{d}\right) \quad \sin \left(\psi_{d}\right) \\
\dot{y}_{d}=u_{d} \sin \left(\psi_{d}-v_{d}\right) \quad \sin \left(\psi_{d}\right) \\
\dot{\psi}_{d}=r_{d} \\
m_{1} \dot{\psi}_{d}-m v_{2} v_{2 t} r_{d}+d u_{d 1}=\tau_{d}+{ }_{1} f \\
m_{2} \dot{v}_{d}+m \mu_{t d} r_{d}+d \quad v_{2 l 2}=0 \\
m_{3} \dot{r}_{d}+m u_{2 d} v_{d}-m u_{d d} \psi_{d}+d \quad r_{d}=\tau_{d}+T_{3}
\end{array}\right.
$$

Define the position tracking error for USV:

$$
\left\{\begin{array}{l}
u_{e}=u-u_{d} \\
v_{e}=v-v_{d} \\
r_{e}=r-r_{d}
\end{array}\right.
$$

Differentiate the function, the error equation: 


$$
\left\{\begin{aligned}
\dot{u}_{e}= & \frac{m_{22}}{m_{11}}\left(v_{e} r_{e}+v_{e} r_{d}+v_{d} r_{e}\right)-\frac{d_{1}}{m_{11}} u_{e} \\
& +\frac{1}{m_{11}}\left(\tau_{1}-\tau_{1 d}\right) \\
\dot{v}_{e}= & -\frac{m_{11}}{m_{22}}\left(u_{e} r_{d}+u_{e} r_{e}+u_{d} r_{e}\right)-\frac{d_{22}}{m_{22}} v_{e} \\
\dot{r}_{e}= & \frac{m_{11}-m_{22}}{m_{33}}\left(v_{e} u_{e}+u_{e} v_{d}+v_{e} u_{d}\right)-\frac{d_{33}}{m_{33}} r_{e} \\
& +\frac{1}{m_{33}}\left(\tau_{3}-\tau_{3 d}\right)
\end{aligned}\right.
$$

Design first-order exponential stable sliding mode switching surface:

$$
S_{1}=u_{e}+\lambda_{1} \int_{0}^{t} u_{e}(\tau) d \tau, \quad \lambda_{1}>0
$$

Differentiate the switching surface $S_{1}$ :

$$
\begin{aligned}
\dot{S}_{1} & =\dot{u}_{e}+\lambda_{1} u_{e} \\
& =\lambda_{1} u_{e}-\dot{u}_{d}+\frac{\tau_{1 d}+f+m_{22} v_{d} r_{d}-d_{11} u_{d}}{m_{11}}
\end{aligned}
$$

Make $\dot{S}_{1}=0$, the equivalent control law is obtained:

$$
u_{e q}=\hat{d}_{11} u_{d}-\hat{m}_{11}\left(\lambda_{1} u_{e}-\dot{u}_{d}\right)-\hat{m}_{22} v_{d} r_{d}
$$

The "^" indicates the estimated value of uncertain model parameters, the same below.

In order to eliminate the phenomenon of "high frequency flutter" of the system, this paper adopts saturation function $\operatorname{sat}\left(S_{1} / \phi_{1}\right)$ to replace the function of the desired sliding mode $\operatorname{sgn}\left(S_{1}\right)$.

$$
\operatorname{sat}\left(S_{1} / \partial\right)= \begin{cases}S_{1} / \partial, & \left|S_{1}\right| \leq \partial \\ \operatorname{sgn}\left(S_{1}\right), & \left|S_{1}\right|>\partial\end{cases}
$$

Define switching control law:

$$
\mathrm{u}_{s w}=\zeta_{1} \cdot \operatorname{sat}\left(S_{1} / \partial\right)
$$

Therefore, the longitudinal driving force control law of water jet propulsion USV with parameter uncertainty and external disturbance is designed:

$$
\begin{aligned}
f= & \hat{d}_{11} u_{d}-\hat{m}_{11}\left(\lambda_{1} u_{e}-\dot{u}_{d}\right) \\
& -\hat{m}_{22} v_{d} r_{d}-\zeta_{1} \cdot \operatorname{sat}\left(S_{1} / \partial\right)
\end{aligned}
$$

In order to determine the parameters of the state function of the USV, define the boundaries of hydrodynamic damping coefficient and inertia parameter which includes additional mass as :

$$
\left|m_{i i}-\hat{m}_{i i}\right| \leq M_{i i} \quad\left|d_{i i}-\hat{d}_{i i}\right| \leq D_{i i}, \quad i=1,2,3 .
$$

Select the following Lyapunov function:

$$
V_{1}=\frac{1}{2} m_{11} S_{1}^{2}
$$

Differentiate the function and calculate $\dot{S}_{1} 、 f$ in the formula:

$$
\begin{aligned}
\dot{V}_{1} & =m_{11} S_{1} \dot{S}_{1} \\
& =S_{1}\left\{\begin{array}{l}
\left(\lambda_{1} u_{e}-\dot{u}_{d}\right)\left(m_{11}-\hat{m}_{11}\right)+v_{d} r_{d}\left(m_{22}-\hat{m}_{22}\right) \\
-u_{d}\left(d_{11}-\hat{d}_{11}\right)-\zeta_{1} \cdot \operatorname{sat}\left(S_{1} / \partial\right)
\end{array}\right\}
\end{aligned}
$$

According to the condition of sliding, the formula(16) must be satisfied[8]:

$$
\dot{V}_{1}=m_{11} S_{1} \dot{S}_{1} \leq-\eta_{1} \hat{m}_{11}\left|S_{1}\right|, \quad \eta_{1}>0
$$

Combine formula (16) and (17), state parameter ${ }^{7} 1$ is solved:

$$
\begin{aligned}
\vec{\supset}_{1}= & M_{11}\left|\lambda_{1} u_{e}\right|+M_{11}\left|\dot{u}_{d}\right|+M_{22}\left|v_{d} r_{d}\right| \\
& +D_{11}|u|+m_{11} \eta_{1}
\end{aligned}
$$

Design second-order exponential stable sliding mode switching surface:

$$
S_{2}=\dot{v}_{e}+2 \lambda_{2} v_{e}+\lambda_{2} \int_{0}^{t} v_{e}(\tau) d \tau, \quad \lambda_{2}>0
$$

Differentiate the switching surface $S_{2}$ :

$$
\dot{S}_{2}=\ddot{v}_{e}+2 \lambda_{2} \dot{v}_{e}+\lambda_{2}{ }^{2} v_{e}
$$

Differentiate the second formula of (8):

$$
\begin{aligned}
\ddot{v}_{e}= & -\frac{m_{11}}{m_{22}}\left(\begin{array}{l}
\dot{u}_{e} r_{d}+u_{e} \dot{r}_{d}+\dot{u}_{e} r_{e} \\
+u_{e} \dot{r}_{e}+u_{d} \dot{r}_{e}+\dot{u}_{d} r_{e}
\end{array}\right)-\frac{d_{22}}{m_{22}} \dot{v}_{e} \\
= & -\frac{m_{11}}{m_{22}}\left(u_{e}+u_{d}\right) \dot{r}_{e} \\
& -\frac{m_{11}}{m_{22}}\left(\begin{array}{l}
\dot{u}_{e} r_{d}+u_{e} \dot{r}_{d} \\
+\dot{u}_{e} r_{e}+\dot{u}_{d} r_{e}
\end{array}\right)-\frac{d_{22}}{m_{22}} \dot{v}_{e}
\end{aligned}
$$

Make:

$$
Q=-\frac{m_{11}}{m_{22}}\left(\dot{u}_{e} r_{d}+u \dot{r}_{e}+\dot{u} r_{e}+\dot{u} r\right)-\frac{d}{m_{22}} \dot{v}
$$




$$
\begin{aligned}
\dot{S}_{2}= & Q-\frac{m_{11}}{m_{22}}\left(u_{e}+u_{d}\right) \dot{r}_{e}+2 \lambda_{2} \dot{v}_{e}+\lambda_{2}{ }^{2} v_{e} \\
= & Q-\frac{m_{11}}{m_{22}}\left(u_{e}+u_{d}\right)\left\{\begin{array}{l}
\frac{m_{11}-m_{22}}{m_{33}}\left(\begin{array}{c}
v_{e} u_{e}+ \\
u_{e} v_{d}+v_{e} u_{d}
\end{array}\right) \\
-\frac{d_{33}}{m_{33}} r_{e}+\frac{1}{m_{33}}\left(\tau_{3}-\tau_{3 d}\right)
\end{array}\right\} \\
& +2 \lambda_{2} \dot{v}_{e}+\lambda_{2}{ }^{2} v_{e}
\end{aligned}
$$

Make:

$$
\begin{gathered}
W=Q m_{22} m_{33}-m_{11}\left(u_{e}+u_{d}\right)\left\{\begin{array}{c}
\left(m_{11}-m_{22}\right)\left(\begin{array}{c}
v_{e} u_{e} \\
+u_{e} v_{d}+v_{e} u_{d}
\end{array}\right. \\
-d_{33} r_{e}+\left(\tau_{3}-\tau_{3 d}\right)
\end{array}\right\} \\
+m_{22} m_{33}\left(2 \lambda_{2} \dot{v}_{e}+\lambda_{2}^{2} v_{e}\right) \\
N=m_{11}\left(u_{e}+u_{d}\right)
\end{gathered}
$$

$\dot{S}_{2}=0$, the equivalent control law is obtained:

$$
\hat{T}_{e q}=\frac{\hat{W}}{\hat{N}}
$$

$\hat{W}$ and $\hat{N}$ are estimated value. So, the yaw torque tracking control law for USV is designed:

$$
T=\frac{\hat{W} \zeta_{2} \cdot \operatorname{sat}\left(S_{2} / \partial_{2}\right)}{\hat{N}}
$$

When $\hat{N}=0$, the control law produces a singular value. we can assume $\hat{N}>0$.

Similar to the literature $[9,10]$, assume the parameter $h$ is limited, $|h-\hat{h}| \leq H$, select the following Lyapunov function:

$$
V_{2}=\frac{1}{2} m_{22} m_{33} S_{2}^{2}
$$

Differentiate the Lyapunov function:

$$
\begin{aligned}
& \dot{V}_{2}=m_{2} m S_{3} \dot{S}_{2} \quad 2 \\
& =S_{2}\left\{\left(N \cdot \frac{\hat{W} \zeta_{2} \cdot \mathrm{s} \mathrm{a} \mathrm{tS}\left({ }_{2} \partial /{ }_{2}\right.}{\hat{N}}\right)-W\right\} \\
& =S_{2}\left\{\begin{array}{l}
\left(N \hat{N}^{-1}-1\right) \hat{W}+\hat{W} \\
-W-N \hat{N}^{-} \zeta_{2} \cdot \operatorname{sat}\left(S_{2} / \partial\right)
\end{array}\right\}
\end{aligned}
$$

Define geometric mean of parameters $N$ :

$$
\left\{\begin{array}{l}
N_{\max }=\left(\hat{m}_{22}+M_{22}\right) u_{d}-\left(\hat{m}_{11}-M_{11}\right) u>0 \\
N_{\min }=\left(\hat{m}_{22}-M_{22}\right) u_{d}-\left(\hat{m}_{11}+M_{11}\right) u>0 \\
\hat{N}=\sqrt{N_{\max } N_{\min }} \\
\beta=N_{\max } / N_{\min } \\
1 / \beta \leq N \hat{N}^{-1} \leq \beta
\end{array}\right.
$$

If design control parameters $\partial_{2}$ as follows:

$$
\partial_{2}=\beta\left(H+\hat{m}_{22} \hat{m}_{33} \eta_{2}\right)+(\beta-1)|\hat{W}|
$$

So

$$
\dot{V}_{2}=S_{2}\left\{\begin{array}{l}
\left(N \hat{N}^{-1}-1\right) \hat{W}+\hat{W} \\
-W-N \hat{N}^{-1}\left(\beta\left(H+\hat{m}_{22} \hat{m}_{33} \eta_{2}\right)\right. \\
+(\beta-1)|\hat{W}|) \cdot \operatorname{sat}\left(S_{2} / \partial_{2}\right)
\end{array}\right\}
$$

From the above, $N \hat{N}^{-1} \beta \geq 1$, so:

$$
\left\{\begin{array}{l}
-N \hat{N}^{-1} \beta H \cdot \text { s a t }\left(S_{2} \partial{ }_{2} \cdot S_{2}\right)+\hat{W}-\left(W \leq S_{2}\right) \\
-N \hat{N}^{-1} \beta \hat{m}_{22} \hat{m}_{33} \eta_{2} \cdot \text { s a t }\left(S_{2} \partial /_{2} \cdot S_{2}\right) \leq 0 \\
-N \hat{N}^{-1}(\beta-1|\hat{W}|) \cdot \operatorname{sat}\left(S_{2} \partial l_{2} \cdot S_{2}\right) \\
+\left(N \hat{N}^{-1}-1\right) \hat{W} S_{2} \leq 0
\end{array}\right.
$$

So

$$
\dot{V}_{2}=m_{22} m_{33} S_{2} \dot{S}_{2} \leq-\hat{m}_{22} \hat{m}_{33} \eta_{2}\left|S_{2}\right|
$$

That satisfies the sliding condition.

\subsection{Stability analysis}

The above design control laws satisfy the sliding mode condition, and the selected two switching surface are asymptotically stable. Therefore, the trajectory of USV approach to the switching surface with exponential function. In order to prove the stability of USV systems, often by proving the yaw motion is bounded input bounded output stability (BIBO)[11]. Define the following Lyapunov function:

$$
V_{3}=\frac{1}{2} m_{33} r^{2}
$$

Derivative to the time:

$$
\dot{V}_{3}=r\left(T+\tau_{3}-d_{33} r+m_{11} u v-m_{22} u v\right)
$$

When the function $V_{3}$ is a decreasing function, $r$ must be a decreasing function. Accordingly, we can know that when $d_{33}|r|>\left|T+\tau_{3}-m_{d} u v\right|, \quad \dot{V}_{3}<0, \quad$ and 
because the variables $T 、 \tau_{3} 、 u 、 v$ are bounded, so $r$ must be a bounded value.

In summary, the USV's yaw motion satisfies the BIBO characteristics.

\section{Simulation experiment}

In order to verity and illustrate the performance of the control schemes proposed for trajectory tracking control of underactuated USV, computer simulations were carried out on an USV developed by Shanghai University, simulation environment is a tool platform based on Matlab/Simulink.

The simulation experiment considers the contrast test under the condition that the USV exists model parameters perturbation and environmental disturbance. In this paper, the linear trajectory and circular trajectory are simulated and tested in the case of external disturbance and non-disturbance, and the validity and feasibility of the controller are verified by experiments.

Fig. 2 and Fig. 3 are USV 's simulation results for linear trajectory tracking(Red represents the desired trajectory of the USV, black for the actual trajectory, green ship type represents the direction of the ship's bow, the same bellow), the desired trajectory of the USV is a straight line with a slope of 0.02 , its equation is:

$$
x_{d}=0.5+0.02 t, \quad y_{d}=0.5+0.02 t
$$

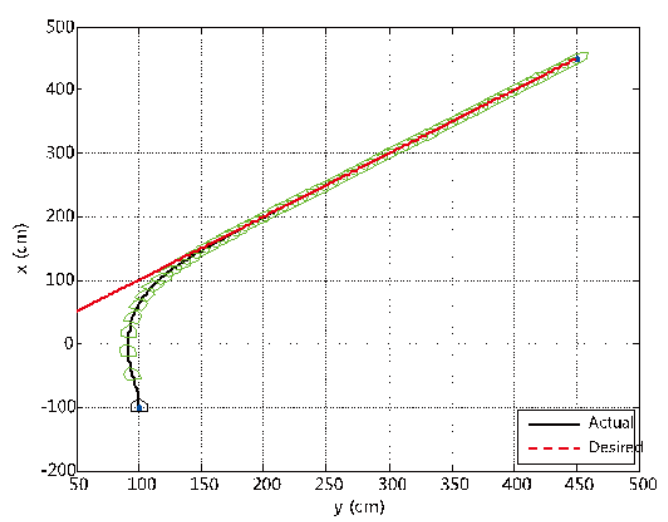

Figure 2. Desired linear trajectory and actual trajectory without interference.
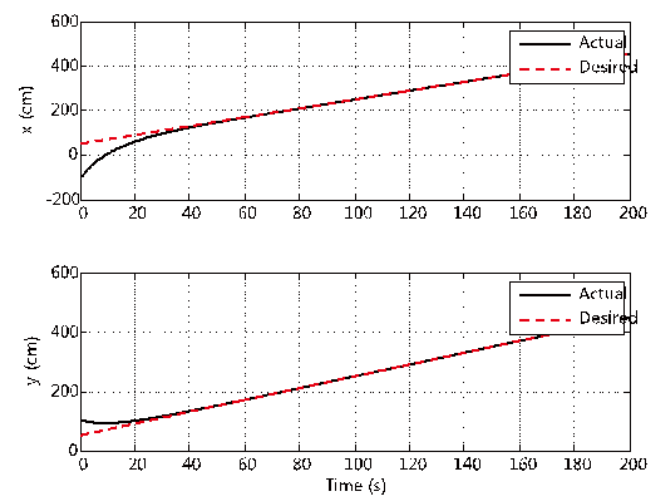

Figure 3. Desired position and actual position without interference.
Fig. 4 and Fig. 5 are USV 's simulation results for circular trajectory tracking, the desired trajectory of the USV is a circle with a radius of 2 , its equation is:

$$
x_{d}=2 \cos (\pi / t), \quad y_{d}=2 \sin (\pi / t) .
$$

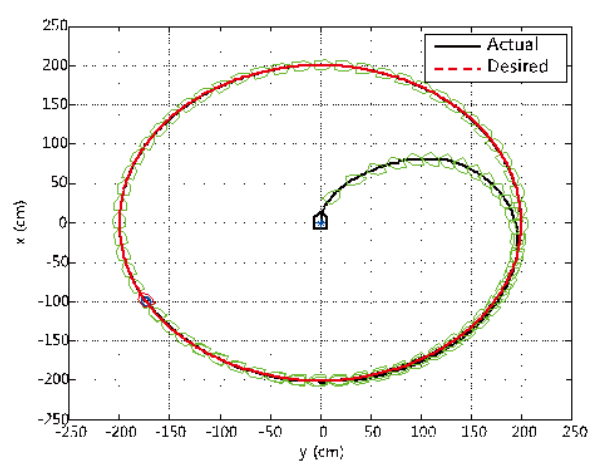

Figure 4. Desired circular trajectory and actual trajectory without interference.
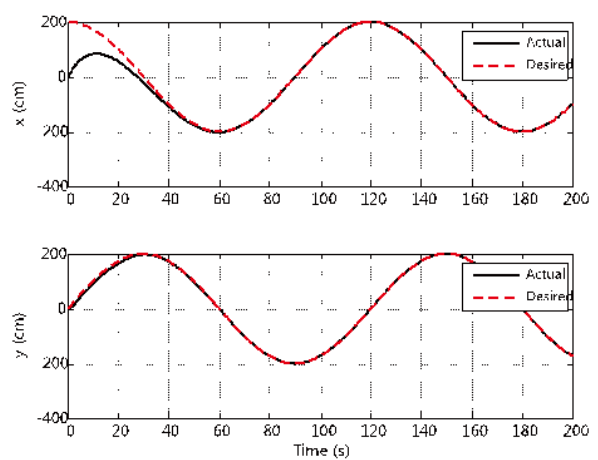

Figure 5. Desired position and actual position without interference.

From the results of the simulation test in Fig. 4 and Fig. 5, in the absence of external disturbance, USV not only can quickly track the desired trajectory in position, the direction of the ship's bow also can point to the tangential direction of movement correctly. To further verify the advantages of the controller designed in this paper, comparing the trajectory tracking of the circular motion under the condition of interference and non-interference. The overall effect of the trajectory tracking simulation under the conditions of environmental interference is shown in Fig. 6, and desired position and actual position with interference is shown in Fig. 7.

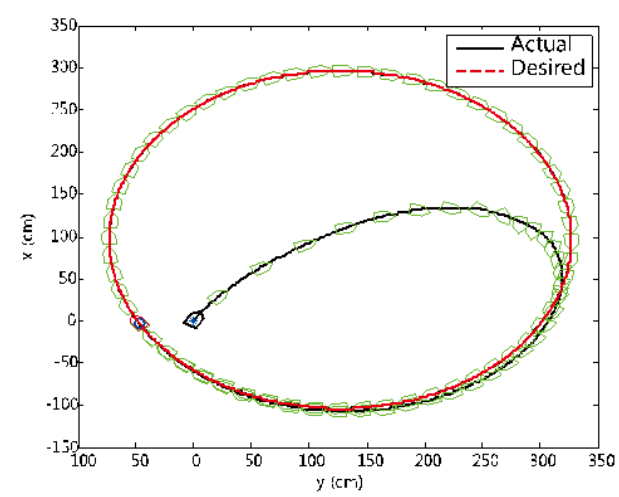

Figure 6. Desired circular trajectory and actual trajectory with interference. 

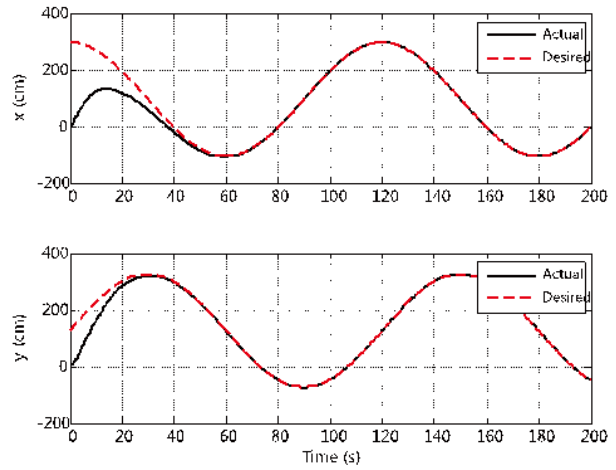

Figure 7. Desired position and actual position with interference.

Fig. 8 and Fig. 9 show response curves of surge velocity and sway velocity of USV under different conditions. it is suggested that the controller has a smooth tracking control for the desired trajectory.
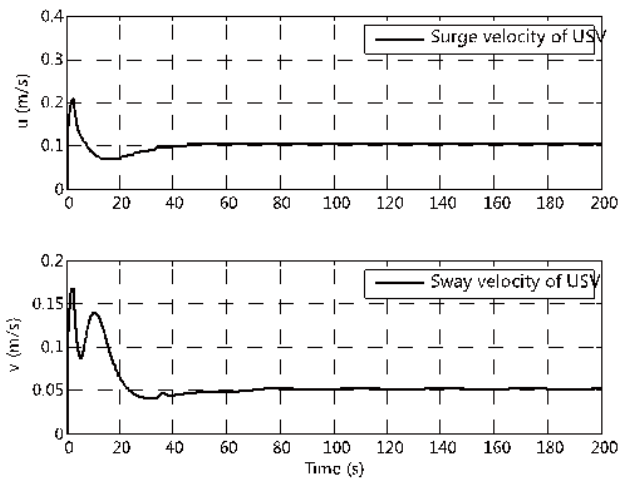

Figure 8. Surge and sway velocity with non-interference .
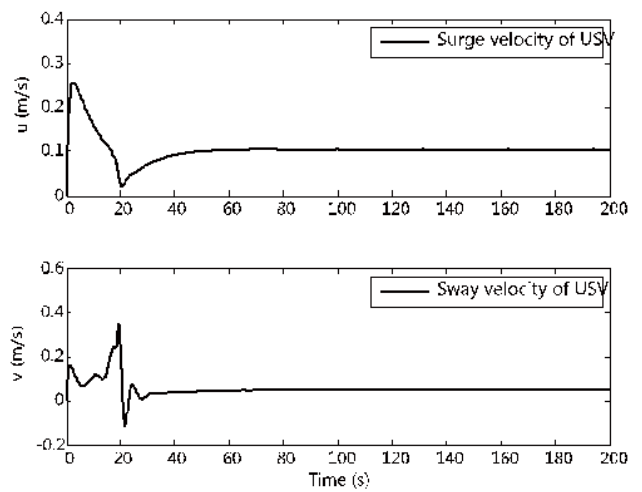

Figure 9. Surge and sway velocity with interference .

Simulation results in Figs. 2 to 9 show that:

1. The control algorithm proposed could achieve trajectory tracking control of USV without the role of side thrusters.

2. The controller guarantees that the USV can track a circle trajectory and a straight line trajectory and have the actual trajectory rapidly convergence to desired trajectory.

3. Global robustness and precise control performance of the controller designed is shown in the simulation experiment results.

\section{Conclusions}

An improved trajectory tracking controller is proposed in this paper for trajectory tracking control of underactuated USV, based on backstepping control algorithm, combined with sliding mode control method. This controller can track both straight line trajectory and curve trajectory with relatively high accuracy. The stability of USV system is proved by BIBO characteristics. and the control algorithm is verified and illustrated in simulation experiments.

\section{References}

1. Godhavn, J.M. Nonlinear tracking of underactuated surface vessels[C]. In proceedings of the $35^{\text {th }}$ IEEE Conference on Decision and Control kobe,Japan. Dec. 1996, Vol. 1: 975-980.

2. Pettersen, K.Y., Nijmeijer H. Tracking Control of an Underactuated Surface Vessel[C]. Proceedings of the IEEE Conference on Decision and Control, Tampa, Florida, 1998: 4561-4566.

3. Do, K.D., Jiang, Z.P., Pan, J. Underactuated ship global tracking under relaxed conditions[J]. IEEE Transactions on Automatic Control, 2002, 47(9): 1529-1536.

4. Aguiar, A.P., Hespanha, J.P. Logic-based switching control for trajectory-tracking and path-following of underactuated autonomous vehicles with parametric modeling uncertainty[C]. Proceedings of the 2004 American Control Conference, Boston, USA, 2004: 3004-3010P.

5. Yan P, Jianda H, Qi S. Tracking Control of Underactuated Surface Ships: Using Unscented Kalman Filter to Estimate the Uncertain Parameters[C]. Proceedings of the 2007 IEEE International Conference on Mechatronics and Automation Harbin, China. 2007:1884-1889.

6. Do, K.D., Pan, J. State and output-feedback robust path-following controllers for underactuated ships using Serret-Frenet frame[J]. Ocean Engineering. 2004, 31: 587-613P.

7. WAN Lei, CUI Shipeng, ZHANG Guocheng, et al . Path following control of underactuated autonomous underwater vehicles $[\mathrm{J}]$. Electric Machines and Control, 2013, 17( 2) : 103-111.

8. J.J.E Slotine. Application of nonlinear control [M]. Beijing: press of mechanical industry, 2009.

9. Ashrafiuon $\mathrm{H}$, Muske $\mathrm{K}$. Sliding mode tracking control of surface vessels[C]. Seattle,Washington, USA, 2008: 556-561.

10. Ashrafiuon H, Muske K, Mcninch L, et al. Sliding mode tracking control of surface vessels[J]. IEEE Transactions on Industrial Electronics. 2008, 55(11): 4004-4012.

11. Mcninch L, Ashrafiuon H, Muske K. Optimal specification of sliding mode control parameters for unmanned surface vessel systems[C]. Proceedings of the 2009 American Control Conference, Hyatt Regency Riverfront, USA, 2009: 2350-2355. 